\title{
Pyoderma gangrenosum: An ulcerative variant developing at a paraincisional LSCS scar site
}

\author{
Kritika Pandey ${ }^{1}$, Mansak Shishak², Neeraj Yadav ${ }^{3}$
}

\author{
${ }^{1}$ Consultant Dermatologist, Skin Clinic, Haryana, India, ${ }^{2}$ Department of Dermatology, Indian Spinal Injuries Centre, New \\ Delhi, India, ${ }^{3}$ Consultant Gynaecologist, Rewari, Haryana, India
}

Corresponding author: Dr. Mansak Shishak, E-mail: mansakshishak@gmail.com

\begin{abstract}
Pyoderma gangrenosum (PG) is an uncommon entity based on a diagnosis of exclusion. It can manifest itself mimicking various ulcerative cutaneous conditions and nonhealing wounds. With its less known etiology and presentation, there is a danger of mistreatment. Herein, we present an interesting case of pyoderma gangrenosum in a young woman, developing around the area of a lower segment Cesarean scar, but not involving the scar tissue. The PG was not associated with any underlying systemic ailments and responded well to a tapering dose of oral corticosteroids. Prompt diagnosis and initiation of therapy lead to a good response and favorable prognosis. Recognizing the atypical clinical presentations of PG is pertinent. Treatment with immunosuppressive agents and a multidisciplinary approach are recommended.
\end{abstract}

Key words: Ulcer; Pyoderma gangrenosum; LSCS; Neutrophilic dermatosis

\section{INTRODUCTION}

Pyoderma gangrenosum (PG) is an inflammatory, noninfectious neutrophilic dermatosis usually characterized by rapidly developing ulcers, first described by Brunsting et al. [1]. PG is an uncommon entity, frequently associated with underlying conditions, such as inflammatory bowel disease, polyarthritis, and hematologic and hepatic disorders. One of its causes might be a dysregulation of the immune system. PG is rarely associated with pregnancy and surgical scar sites, and its appearance in areas around surgical scars, likewise, has not been reported in the literature.

\section{CASE REPORT}

A 25-year-old $\mathrm{G}_{1} \mathrm{P}_{1}$ woman with no history of underlying systemic ailments presented with painful, sudden-onset ulcerations, associated with swelling and induration. Twenty days prior, she underwent a lower segment Cesarean section (LSCS), which was seemingly uneventful. However, nine days after the operation, she developed painful reddish papules on the lower abdomen. Initially small and few, they increased in size rapidly over several days and started forming ulcers (Fig. 1). The patient was anxious and in visible distress due to the burning pain at the ulcer sites. Upon examination, the ulcers were multiple, tender, with undermined edges and mild violaceous changes at the borders. They were present on either side of the Cesarean wound. The surgical scar, however, had healed well with no signs of dehiscence. She was otherwise afebrile with stable vitals and no lymphadenopathy on palpation.

Routine blood investigations, including acute phase reactants (ESR and CRP), complete blood count, APLA and RA factor, were within normal limits. Viral markers were nonreactive. A pus culture was sterile.

A presumptive diagnosis of pyoderma gangrenosum was reached after excluding other infectious and autoinflammatory conditions.

A skin biopsy was taken from the margin of the ulcer, and histopathology showed neutrophilic infiltrate in the dermis and mild necrosis of the overlying epidermis,

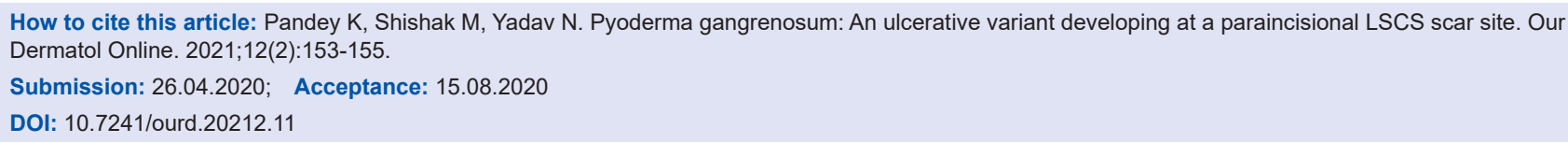


with acute and chronic inflammatory features. In the absence of other classical features suggestive of vasculitis and granuloma, a diagnosis of pyoderma gangrenosum was established

She was counseled and started on oral prednisolone (l mg per kg of body weight), an oral antibiotic course of doxycycline $100 \mathrm{mg}$ twice daily, and anti-inflammatory agents twice daily. The patient was advised to do wound care. Topical tacrolimus and mupirocin application to the ulcers were prescribed. Within a month of initiating treatment, the ulcers reduced in size by $50 \%$ along with a decrease in pain and discomfort. After a three-month course of tapering steroids, there was a significant and dramatic improvement (Fig. 2). All ulcers healed with scarring and the patient reported an excellent general health condition.

\section{DISCUSSION}

Pyoderma gangrenosum commonly occurs in the lower extremities, displays a male predominance, and has rarely

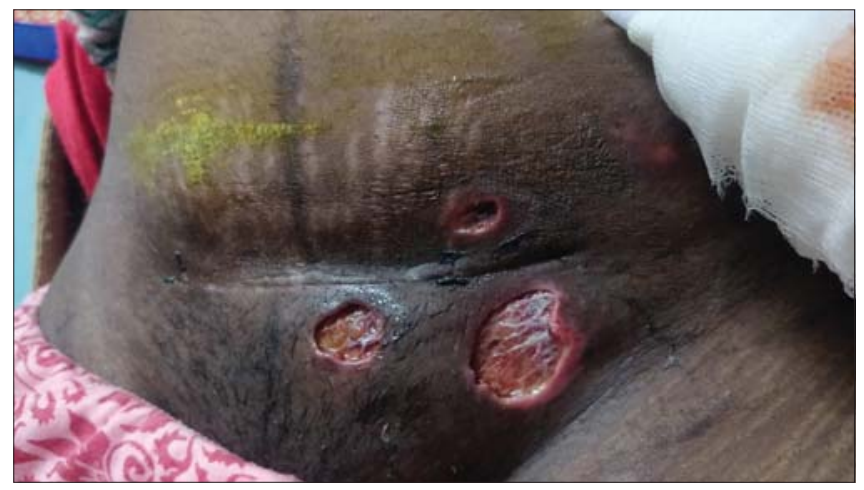

Figure 1: Ulcerations with indurated, inflammatory, and violaceous margins.

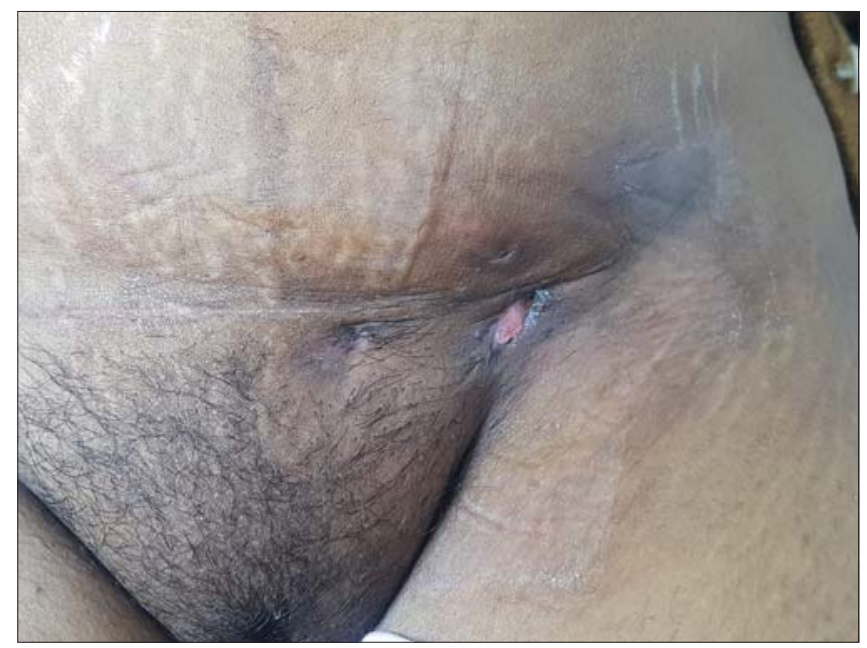

Figure 2: Clean and well-healed scars after three months of therapy. been reported to complicate Cesarean sections [2]. Its exact etiology is yet unknown but resembles class II Schwartzman-like hypersensitivity reactions. Initially a single pustular lesion, it is known to progress rapidly, causing painful ulcerated nodules or plaques with undermined, dusky, and violaceous borders that heal with cribriform scarring [3]. Though commonly associated with other inflammatory, infectious, and malignant pathologies, it may at times be idiopathic with no relevant causative factors [4]. While, in adults, it is found in up to $74 \%$ cases, in children, it is less common, with only a handful of reported cases $(50 \%$ of cases) [5]. The underlying systemic predisposing factors include ulcerative colitis, Crohn's disease, arthritis, hematological malignancies, HIV, and lupus.

The chances of developing PG during pregnancy and postpartum, albeit rare, may occur due to immunosuppression. Alterations of immune function in pregnant women could play a role in the development of PG during pregnancy due to IL-2 and IL-1 inhibition and depressed polymorphonuclear leukocyte (PMN) chemotaxis and adherence functions. An increase in granulocyte-macrophage colony-stimulating factor, a known attractant of neutrophilic inflammation, and an increase in band neutrophils could possibly magnify the risk of neutrophil-driven PG [6]. Histology is nonspecific, which makes PG predominantly a clinical diagnosis of exclusion. Histopathology shows dermal neutrophilic infiltrate in $50 \%$ of cases [7].

There have been few reports of cases of post-surgery pyoderma gangrenosum, leading to delay in diagnosis. Additionally, post-surgical cases wherein lesions develop at the site of the suture wound itself are attributed to the pathergy reaction [8].

Our patient, however, had no underlying medical conditions and no history suggestive of an autoimmune diathesis or an immunosuppressive state. However, the patient met enough criteria-five out of eight- to be diagnosed with PG: 1) exclusion of infection; 2) a history of papules, pustules, or vesicles ulcerating within four days of appearing; 3) peripheral erythema, an undermining border, and tenderness at the ulceration site; 4) a cribriform or "wrinkled-paper" scar at healed ulcer sites; and 5) a decrease in the size of the ulcer within a month of initiating the immunosuppressive medication of corticosteroids [9].

What is special in this case is the development of lesions only around the scar site, but not affecting the 
scar tissue itself, which is unlike the existing cases in the literature. To the best of our knowledge, such a case has not been documented in the literature. The question remains whether this was a coincidental occurrence or whether there is a possibility of development in a paraincisional area without trauma, in which case the inciting cause cannot be determined exactly.

\section{CONCLUSION}

Pyoderma gangrenosum is a rare entity, especially in the setting of pregnancy and surgery. It is likely to be misdiagnosed as a case of complicated wound healing, leading to a poor response to wound care, such as antibiotics. Prompt and high-index clinical suspicion is needed, followed by quick treatment with immunosuppressive agents. A multidisciplinary approach is recommended for a smooth treatment and alleviation of the patient's symptoms.

\section{Consent}

The examination of the patient was conducted according to the principles of the Declaration of Helsinki.

The authors certify that they have obtained all appropriate patient consent forms, in which the patients gave their consent for images and other clinical information to be included in the journal. The patients understand that their names and initials will not be published and due effort will be made to conceal their identity, but that anonymity cannot be guaranteed.

\section{REFERENCES}

1. Brunsting LA, Goeckerman WH, O'Leary PA. Pyoderma (ecthyma) gangrenosum: clinical and experimental observations in five cases occurring in adults. Arch Dermatol Syphilol. 1930;22:655-80.

2. Incel Uysal P, Gur Aksoy G, Yalcin B. Clinical findings and outcomes in patients with pyoderma gangrenosum: a single tertiary centre experience. Our Dermatol Online. 2019;10:17-22.

3. Abreu Velez AM, Howard MS, Brown VM. Immunoreactivity to dermal vessels in a patient with pyoderma gangrenosum. Our Dermatol Online. 2014;5:419-22.

4. Lamouaffaq A, Gallouj S, Baybay H, Mernissi FZ. Idiopathic pyoderma gangrenosum. Our Dermatol Online. 2019;10:306-7.

5. El Anzi O, Sqalli A, Maouni S, Znati K, Meziane M, Ismaili N, et al. Unusual presentation of pyoderma gangrenosum in an infant. Our Dermatol Online. 2019;10:308-9.

6. Steele RB, Nugent WH, Braswell SF, Frisch S, Ferrell J, OrtegaLoayza AG. Pyoderma gangrenosum and pregnancy: an example of abnormal inflammation and challenging treatment. Br J Dermatol. 2016;174:77-87.

7. Diatta BA, Toumbou FF, Diop A, Diadie S, Ndiaye M, Diallo M, et al. Pyoderma gangrenosum among children in Senegal: 6 cases. Our Dermatol Online. 2017;8:463-6.

8. Zuo KJ, Fung E, Tredget EE, Lin AN. A systematic review of post-surgical pyoderma gangrenosum: identification of risk factors and proposed management strategy. J Plast Reconstr Aesthet Surg. 2015;68:295-303.

9. Maverakis E, Ma C, Shinkai K, Fiorentino D, Callen JP, Wollina U, et al. Diagnostic criteria of ulcerative pyoderma gangrenosum: a Delphi consensus of international experts. JAMA Dermatol. 2018;154:461-6.

Copyright by Kritika Pandey, et al. This is an open-access article distributed under the terms of the Creative Commons Attribution License, which permits unrestricted use, distribution, and reproduction in any medium, provided the original author and source are credited.

Source of Support: Nil, Conflict of Interest: None declared. 